Med Klin Intensivmed Notfmed 2015.

110:108-109

DOI 10.1007/s00063-015-0004-4

Online publiziert: 25. März 2015

๑) Springer-Verlag Berlin Heidelberg 2015

\title{
G. Marx
}

Klinik für Operative Intensivmedizin und Intermediate Care, Universitätsklinikum RWTH Aachen, Aachen, Deutschland

\section{Neue AWMF-S3-Leitlinie Volumentherapie}

\section{Auf Wiedersehen Emotionen - willkommen Evidenz}

einem sehr arbeitsintensiven, aber immer konsensorientierten Prozess 42 praxisrelevante und in der Praxis anwendbare evidenzbasierte Empfehlungen und 6 Statements über die Volumentherapie zu verabschieden [1].

Da diese Leitlinie die größtmögliche methodische und inhaltliche Qualität erreichen sollte, entschloss sich die DGAI, Methodenexperten zu beauftragen, um mit diesen gemeinsam die Leitlinie zu erstellen. Es gelang, das Institut für Forschung in der Operativen Medizin unter der Leitung von Herrn Prof. Neugebauer für diese Aufgabe zu gewinnen. Jedes einzelne Abstract, jede Publikation und jede Evidenztabelle wurde sowohl von Klinikern als auch Methodenexperten beurteilt und konsentiert - und der Lohn dieses gewaltigen Arbeitsaufwands sind sehr robuste evidenzbasierte Empfehlungen für die Volumentherapie.

Im Rahmen dieses vorliegenden Themenschwerpunkts haben Ihnen Experten, die auch Mitglieder der Leitliniengruppe sind, mit ihren Mitarbeiten die gegenwärtige medizinische Evidenz kompetent und kritisch zusammengefasst. In dem Beitrag von Herrn Janssens und Herrn Kluge werden die wichtigen Themen Diagnose, Indikation und Steuerung behandelt. In der Leitlinie hat dieser Themenkomplex den sehr notwendigen Stellenwert. Unter anderem wird die hochevidente Empfehlung dargestellt, dass man die Messung des zentralvenösen Drucks nicht zur Diagnostik eines Volumenmangels verwenden kann.

Im nächsten Beitrag beschreiben Herr Waydhas und Kollegen sehr präzise den evidenzbasierten Einsatz von Kristalloiden bei Interventionen und Operationen. In diesem Beitrag wird deutlich, dass die Verwendung von 0,9\%iger NaCl-Lösung als kristalloider Volumenersatz obsolet ist.

Die Kollegen Artmann, Gan und Kranke stellen im nächsten Beitrag ausführlich die Indikationen und Limitationen für den Einsatz von Kolloiden bei Interventionen und Operationen dar - ein spannendes Thema mit klaren Handlungsempfehlungen.

Der Einsatz von Kristalloiden und Kolloiden in der Intensivmedizin sind die Themen der beiden letzten Beiträge von Herrn Wildenauer, respektive Herrn Brülls und Herrn Schindler. Auch hier sind jetzt sehr klare Handlungsempfehlungen vorhanden.

Die Erstellung einer S3-Leitlinie ist eine herausfordernde Aufgabe, wesentlich wichtiger und noch herausfordernder ist jedoch die Translation der evidenzbasierten Volumentherapie zum Patienten.

\section{》) Die Translation \\ der evidenzbasierten \\ Volumentherapie zum \\ Patienten ist wichtig}

In der täglichen Praxis liegt es an uns, die Evidenz in die klinische Praxis umzuwandeln und wir müssen dafür vielleicht an der einen oder anderen Stelle unser klinisches Vorgehen modifizieren.

Abschließend möchte ich mich bei allen sehr bedanken, die an der Erstellung der Leitlinie beteiligt waren! 
Ich wünsche eine interessante und aufschlussreiche Lektüre,

Ihr

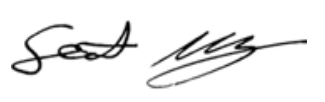

Prof. Dr. Gernot Marx

\begin{tabular}{|l}
\hline Korrespondenzadresse \\
\hline
\end{tabular} $\begin{aligned} & \text { Prof. Dr. G. Marx FRCA } \\
& \text { Klinik für Operative } \\
& \text { Intensivmedizin und } \\
& \text { Intermediate Care } \\
& \text { Universitätsklinikum RWTH } \\
& \text { Aachen, Pauwelsstr. 30 } \\
& \text { 52074 Aachen } \\
& \text { gmarx@ukaachen.de }\end{aligned}$

\section{Einhaltung ethischer Richtlinien}

Interessenkonflikt. G. Marx gibt an, Koordinator der AWMF-S3-Leitlinie „Volumentherapie beim Erwachsenen" zu sein. Er erhielt finanzielle Unterstützung von den Firmen B. Braun Melsungen AG und Serumwerk Bernburg AG für die Durchführung wissenschaftlicher Projekte. Unabhängig hiervon hat er Honorare im Rahmen von Vortragstätigkeiten von diesen Firmen sowie von Fresenius SE \& Co. KGaA und CSL Behring $\mathrm{GmbH}$ erhalten. Er ist Berater von $\mathrm{B}$. Braun Melsungen AG.

Dieser Beitrag beinhaltet keine Studien an Menschen oder Tieren.

\section{Literatur}

1. AWMF (2014) Intravasale Volumentherapie beim Erwachsenen. Leitliniendetailansicht. www.awmf. org/leitlinien/detail/ll/001-020.html. Zugegriffen: 17. Februar 2015

\section{springermedizin.de - komfortabel recherchieren in der e.Bibliothek}

Ohne Umwege zur gewünschten Information zu gelangen - springermedizin.de macht's möglich. Verzichten Sie auf überquellende Stehsammler und unübersichtliche Papierstapel, und nutzen Sie statt dessen die digitale e.Bibliothek von Springer Medizin.

Suchen Sie Beiträge in einer bestimmten Fachzeitschrift Ihres Fachgebiets? Oder möchten Sie englischsprachige Journals für eine interdisziplinäre Recherche nutzen? Interessieren Sie sich für Übersichtsbeiträge oder aktuelle wissenschaftliche Studien?

Die e.Bibliothek wird all diesen Anforderungen gerecht: Sie umfasst über 600 deutschsprachige und internationale Fachzeitschriften aus allen Bereichen der Medizin inklusive der medizinischen Inhalte von SpringerLink.

Die e.Bibliothek beinhaltet auch „Online First"-Beiträge, also hoch aktuelle Beiträge, die bereits vor Erscheinen einer gedruckten Ausgabe in elektronischer Form publiziert wurden.

\section{Komfortable und schnelle Recherche}

- Mit der Volltextsuche von springermedizin.de durchsuchen Sie den gesamten Inhalt der e.Bibliothek und gelangen direkt zu den Inhalten, die für Sie relevant sind.

- Auf Wunsch können Sie die Suchergebnisse eingrenzen und beispielsweise gezielt in einzelnen Zeitschriften, nach Themen und sogar nach Autoren suchen.

- Wenn Sie einen englischen Suchbegriff eingeben, erhalten Sie zudem Treffer aus den englischsprachigen wissenschaftlichen Zeitschriften von SpringerLink.
Mit der Suchfunktion gelangen Sie auch zu Inhalten aus allen anderen Bereichen von springermedizin.de von zertifizierten Fortbildungskursen der e.Akademie, aktuellen Kongressberichten bis hin zu aktuellen Themendossiers, Videos und Nachrichten aus der Gesundheits- und Berufspolitk.

\section{Ihre persönliche Merkliste}

Finden Sie einen Beitrag besonders interessant oder möchten Sie ihn für die spätere Lektüre vormerken? Auf springermedizin.de können Sie ganz einfach Ihre persönliche Merkliste anlegen: ein Klick auf das Symbol "merken" am Beitragsende genügt und die Beiträge erscheinen unter "Meine Merkliste".

Alle Beiträge sind als PDF-Datei im Layout der gedruckten Ausgabe sowie als HTML-Version verfügbar. In der HTMLVersion können Sie die Vorteile der verlinkten Literatur nutzen und direkt zu den zitierten Quellen gelangen.

e.Med - der Zugang zu allen digitalen Inhalten von Springer Medizin

Zugang zu allen Inhalten der e.Bibliothek bekommen Sie mit e.Med. Lernen Sie die Vorzüge dieses umfassenden Angebots kennen und testen Sie e.Med 30 Tage lang kostenlos und unverbindlich unter

\section{www.springermedizin.de/eMed}

Eine erfolgreiche Recherche wünscht Ihnen Ihr Redaktionsteam Fachzeitschriften von Springer Medizin 